\title{
Preparation and Characterization of Mullite-Silica Aerogel Composite Material
}

\author{
Zhaofeng Song*, Haiquan Zhao \\ Chemistry and Chemical Engineering Department, Environmental and Chemical Engineering School, \\ Foshan University, Foshan, China \\ Email: ^270418231@qq.com
}

How to cite this paper: Song, Z.F. and Zhao, H.Q. (2018) Preparation and Characterization of Mullite-Silica Aerogel Composite Material. Open Journal of Organic Polymer Materials, 8, 43-52. https://doi.org/10.4236/ojopm.2018.84004

Received: September 14, 2018

Accepted: October 26, 2018

Published: October 29, 2018

Copyright (c) 2018 by authors and Scientific Research Publishing Inc. This work is licensed under the Creative Commons Attribution International License (CC BY 4.0).

http://creativecommons.org/licenses/by/4.0/

\begin{abstract}
Silica aerogel due to the high specific surface area, nanometer level pores and low density of the micro structure, had excellent heat-insulating properties. But the characteristics of silica aerogel with low density and high voidage resulted in poor mechanical properties. In order to improve the strength of materials and meet the actual needs, in this paper the heat-insulating composite material was made from the silica aerogel and mullite fiber. This kind of composite material mainly contented of mullite fiber, formed macro block structure, that gas gel is coated and filled with mullite fiber. Furthermore, the composite material had good strength and kept excellent thermal stability at $600^{\circ} \mathrm{C}$. In addition the density of composite decreased with the increasing of the gas gel content.
\end{abstract}

\section{Keywords}

Silica, Aerogel, Mullite, Composite Materials

\section{Introduction}

The silica aerogel has the characteristics of excellent heat-insulating performance and low density, so it has broad prospects in the application of space exploration, military industry, chemical industry, building materials and so on [1]. However, due to the high cost of preparing silica aerogel with supercritical drying method, the brittleness and the easy collapsing of silica aerogel owing to absorbing water, the silica aerogel was greatly limited of mass production and the use ranges. The silica aerogel is a pure white solid consisting of $99 \%$ gas hole that is the minimum thermal conductivity in the all solid material [2]. All the time many experts at home and abroad in order to further improve silica aerogel's performance had done many studies, such as the hydrophobic modification and 
enhancing the plate strength that the silica aerogel was added to high strength fiber in gap [3]. At present the preparation method of silica aerogel is collosol-gel which was originally applied by the French chemist. The drying process is one of the important processes in the preparation of the silica aerogel; therefore the selection of appropriate drying methods for the preparation of the gas gel has an important impact [4]. Supercritical drying is the traditional technology of preparing the gas gel, but the process is high cost and is not suitable for mass production. So it is very necessary to study the low cost, non supercritical drying method for the preparation of the gas gel.

The silicic acid sol $(10-20 \mathrm{~nm})$ has large surface area and low viscosity, so mixed with other substances it has better dispersion and permeability. When the silicon solution is in the loss of water, the monomeric silicic acid gradually gathered synthetic polymeric silica gel coating on the surface of -SiO-O-SiO-. Although sodium water glass has defects such as low viscosity, poor water resistance, and poor resistance to high temperature, it can use for filler as skeleton material to improve material properties [5]. Sodium silicate as raw materials has been the development of more than 50 kinds [6]. Polycrystalline mullite, which is seen as a kind of high-temperature resistant material for attention, is a kind of inorganic fiber [7].

The preparation of silica gel is mainly composed of two processes: sol gel process and alcohol gel [8]:

$$
\begin{array}{r}
\mathrm{Si}(\mathrm{OR})_{4}+4 \mathrm{H}_{2} \mathrm{O} \stackrel{\mathrm{PH}=2-5}{\longrightarrow} \mathrm{Si}(\mathrm{OH})_{4}+\mathrm{HOR} \text { (hydrolysis) } \\
n \mathrm{Si}(\mathrm{OH})_{4} \stackrel{393.2 \mathrm{~K}, 3 \mathrm{~h}}{\longrightarrow}\left(\mathrm{SiO}_{2}\right) n+2 n \mathrm{H}_{2} \mathrm{O} \text { (polycondensation) (1)) } \\
\text { (Equation (2)) }
\end{array}
$$

In acidic conditions $(\mathrm{pH}=2-5)$, the hydrolysis rate is rapidder, the reaction system can promote the nucleation reaction containing large amount of silicic monomer. Finally, we get weak cross-linking degree, network low density gel [9].

In this experiment, the raw materials was mixed in the liquid phase, controlled the appropriate reaction temperature and stirring rate, and got wet silica gel [10]. This method is simple and easy to operate. Because of reducing the amount of organic solvent, the equipment cost, the pollution, the method is suitable for mass production [11].

\section{Materials and Method}

\subsection{Raw Materials and Chemical Reagents for Test}

Sodium water glass was industrial pure from Foshan Zhongfa water glass factory. N-hexane was obtained from Shanghai Reagent 1 Factory with analytical reagent. Nitric acid was produced by Foshan zhong cheng lian Chemical Industry Co., Ltd. (analytical reagent). Hexamethyl-disilazane (HMDS) was purchased from Quzhou aokai Chemical Co., Ltd. (industrial pure, China). Deionized water was prepared using a Hitech master system (Hitech Shanghai). Water-based anti-foam agent was purchased from Guangzhou Guan Zhi Chemical Co., Ltd. 
(industrial pure). Polycrystalline mullite fiber was obtained from Deqing ChenYe crystal fiber Co. Ltd (zhejiang province). OP-10 emulsifier (industry pure) was bought from Shanghai Aladdin biochemical Technology Inc. 30\% silicon sol (industry pure) was obtained from Guangzhou Guangzhou Hui-Ze Environmental Protection Technology Co., Ltd.

\subsection{Equipment for Experiment}

Heating magnetic agitator (DF-101S) was produced from Shanghai Branch Sheng Instrument Co., Ltd, with Heat collection and constant temperature; Scanning electron microscope (NovaNano SEM-430) were purchased from Holland PHILPS; Thermal gravimetric analyzer (DTG-60AH) was obtained from NETZSCH-Gerätebau GmbH of Germany.

\subsection{Preparation Process}

1) measure raw materials: sodium water glass $334 \mathrm{~g}$, deionized water $1800 \mathrm{~g}$, HMDS $154.8 \mathrm{~g}$, the hexane $2500 \mathrm{ml}$;

2) raw materials in order poured into the round bottom flask fast, mixed 10 minutes, joined quickly nitric acid $155.1 \mathrm{~g}$ one-time, continued stirring for 20 minutes;

3) water bath heating $55^{\circ} \mathrm{C}$ for 3 hours, after the solution was stratified, the water is discharged from the bottom of the reactor;

4) after drainage, segmented dried: $95^{\circ} \mathrm{C}-120^{\circ} \mathrm{C}-135^{\circ} \mathrm{C}$ (until no longer liquid reflux rise the temperature);

5) at the end of the drying the oven was set to $120^{\circ} \mathrm{C}$ for 3 hours (shown as Figure 1).

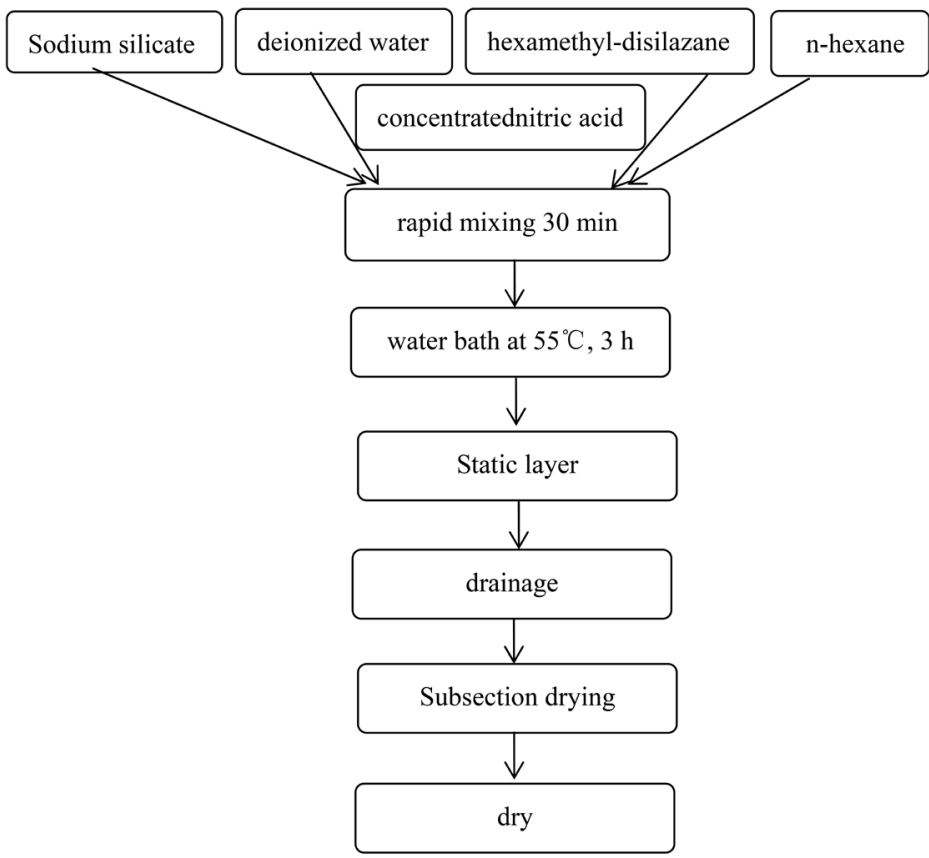

Figure 1. The specific process of preparation of silica gel. 


\subsection{Preparation of Composite Materials}

\subsubsection{Formula Design of Composite Material}

This experiment was using polycrystalline mullite fiber tocreinforce aerogel with silicic acid sol as divergence and bonding agents [12], sodium silicate as a binder. When producing the ratio amount of the raw materials with polycrystalline mullite fiber, silicon sol, sodium water glass, was unchanged, the dosage of silica aerogel changed in order to control the ratio of aerogel in the raw material. Then the slurry was mixed, uniformly lay in the mold, dry in the oven at $120^{\circ} \mathrm{C}$ for 3 hours. Finally we got the insulation composite model that were the content of silica aerogel $0 \%, 20 \%, 30 \%, 40 \%, 50 \%, 60 \%$ respectively.

The polycrystalline mullite fiber as the skeleton of the heat insulation composite material can guarante the forming and strength of the composite to overcome the weaknesses of the aerogel material itself low strength, toughness, unstable. At the same time, the silica aerogels as the filler, the aperture of nano meter level can greatly reduce the effect of heat conduction and heat transfer from gas convection, so that the insulation performance of composite materials has been greatly improved [13].

\subsubsection{Ratio of Raw Materials}

The Silica aerogel has excellent insulation performance but low strength, brittleness, toughness and weaknesses. However the polycrystalline mullite fiber has good mechanical properties [14]. Therefore, the Silica aerogel is necessary polycrystalline Mo mullite fiber to supplement. In order to find out the best balance ratio of thermal insulation and mechanical properties, six group experiments of different percentages of raw materials (Table 1 ) were designed.

\subsubsection{Preparation Process of Polycrystalline Mullite Fiber Composite Material}

1) Preparation of silica gel paste

In order to more easily mix the silica gel with other raw materials, the hydrophobic silica gel was first made in slurry (Figure 2). The $200 \mathrm{ml}$ deionized water was poured into the round bottom flask, then 3 drops of defoaming agent, $3 \mathrm{~g}$ OP-emulsifier, $23 \mathrm{~g}$ aerogel powder was added. The mixture was whisked 3 hours with speed $650 \mathrm{r} / \mathrm{min}$. Finally, the mixture was poured into a beaker to let stand and stratified the water. The $75 \%$ of silica gel slurry was made up [15].

Table 1. Different ratios of raw materials for producting compound material.

\begin{tabular}{ccccccc}
\hline $\begin{array}{c}\text { Raw materials } \\
\text { group }\end{array}$ & 1 & 2 & 3 & 4 & 5 & 6 \\
\hline Mullite fiber cotton/g & 15.05 & 14.98 & 6.20 & 6.10 & 3.21 & 3.12 \\
Silica gel powder/g & 0 & 15.02 & 11.123 & 19.43 & 13.11 & 19.5 \\
Silicic acid sol/g & 24.99 & 25.05 & 10.28 & 10.31 & 5.08 & 5.12 \\
Sodium silicate/g & 25.11 & 25.07 & 10.30 & 10.14 & 5.11 & 5.08 \\
Aerogel content/\% & 0 & 18.20 & 29.34 & 42.26 & 49.45 & 59.41 \\
\hline
\end{tabular}




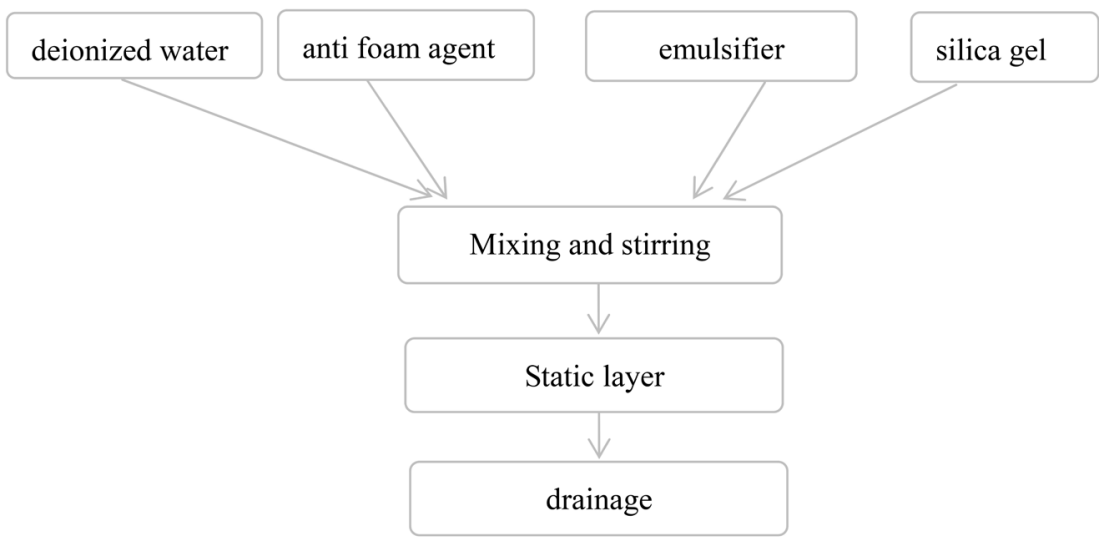

Figure 2. The production process of silica gel slurry.

2) Preparation of thermal insulation composites

According to the proportion of raw materials, the materials were mixed smooth together. Pay attention to control of pulp's consistency, if the slurry is too thin to mold and the slurry is too thick to mix uniformly. The slurry was set on the mold with no a hole or uneven distribution. Then the mold was put into the oven at $120^{\circ} \mathrm{C}$ for 3 hours.

The process of making polycrystalline mullite fiber insulation composite materials was as follows (Figure 3).

\section{Syntheses of the Composite Material}

\subsection{The Microscopic Morphology of Composite Material}

As shown in Figures 4-6, it can be seen that the mullite fibers cross irregular arrangement providing a space for silica aerogel as the basic skeleton of the composite materials. The system shaped under the effect of adhesion in silicic acid sol and sodium water glass and formed composite material. Fiber and silica gel bonded together, so that the composite material has a certain mechanical strength.

\subsection{The Insulation Performance of Composite Material}

The composite material with different content of air gel was put into a closed heating device with a constant temperature $70^{\circ} \mathrm{C}$ for $2 \mathrm{~h}$. The temperature changes of composite materials recorded at regular intervals and the data were plotted on a chart (Figure 7).

As shown in Figure 7, the highest temperature of composite materials decreased with the increase of aerogel content in composite material. From 0 to 3 $\mathrm{min}$, the range of temperature rise was similar. After the $5 \mathrm{~min}$, the composite material with not containing aerogel heated far more than the other. At $18 \mathrm{~min}$, because of different gas gel content in composite materials the temperature varied for each material distinctly. Specifically the higher content materials heated up little instead the lower content materials heated up greatly. 


\subsection{The Thermogravimetric Analysis of Composite Material}

Figure 8 was the TG-DSC curve of the composite material. The thermal experiment was carried out in the air from room temperature to $600^{\circ} \mathrm{C}$ with the speed of $10^{\circ} \mathrm{C} / \mathrm{min}$. From Figure 8 it has been seen that the TG-DSC curve of the composite material has a more obvious endothermic peaks at $71^{\circ} \mathrm{C}$ with $3 \%$ mass loss. The peak was mainly due to the hexane and water absorption volatilization of some remaining in the aerogel. From $200^{\circ} \mathrm{C}$ to $600^{\circ} \mathrm{C}$, the weight loss was only $0.05 \%$ and the DSC curve had no exothermic peak, that is mean the process did not occurre phase transition or ablation. Yet the $0.03 \%$ weight loss was the vaporizein of concluding water in the heating process.

\subsection{The Density Measurement of Composite Material}

As can be seen from Figure 9 and Table 2, the higher content of silica aerogel is, the smaller density of composite material is.

\subsection{The Phase Structure of Composite Material}

Figure 10 was the X-ray diffraction spectrum of composite material (gas gel content $60 \%$ ). From Figure 10 the curve was characterized by diffuse diffraction peaks and the peak intensity was relatively small. The mullite crystal diffraction peak appeared on the dispersion peak. However the mullite crystal diffraction peak was relatively large and big diffraction area.

Table 2. The content and density of Composite material.

\begin{tabular}{cc}
\hline Gas gel content $(\%)$ & Density $\left(\mathrm{g} / \mathrm{cm}^{3}\right)$ \\
\hline 0 & 0.792 \\
29.34 & 0.587 \\
42.7 & 0.461 \\
50 & 0.434 \\
60 & 0.348 \\
\hline
\end{tabular}

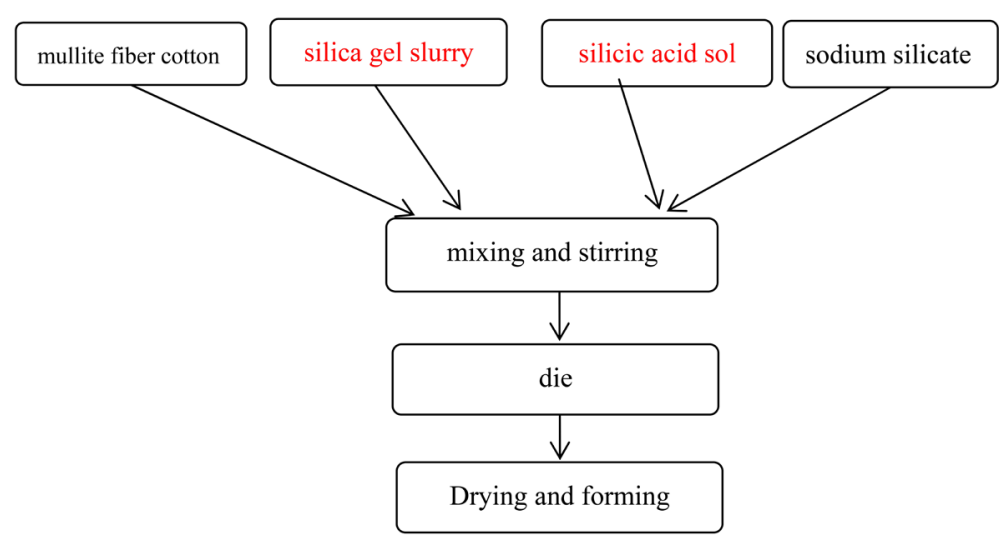

Figure 3. The fabrication process of compound material. 


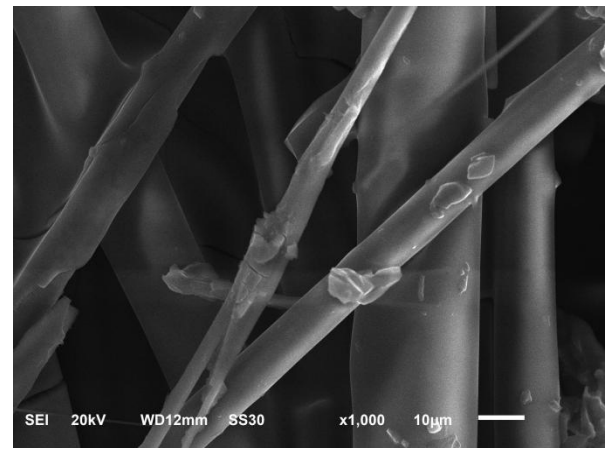

Figure 4. The mullite fiber morphology.

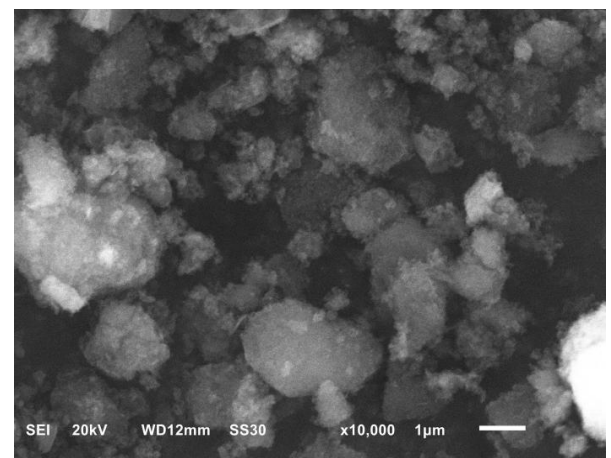

Figure 5. The microstructure of silica gel.

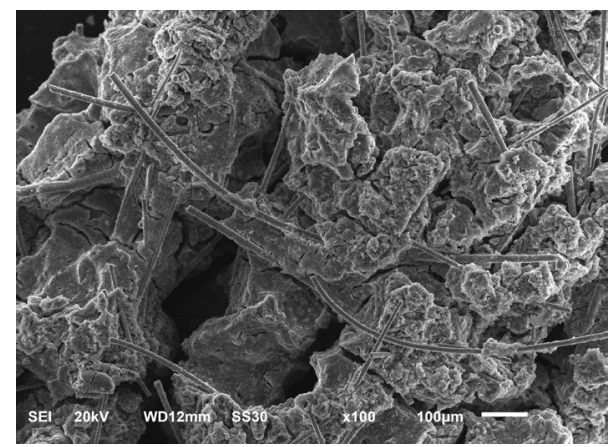

Figure 6. The microstructure of composite material.

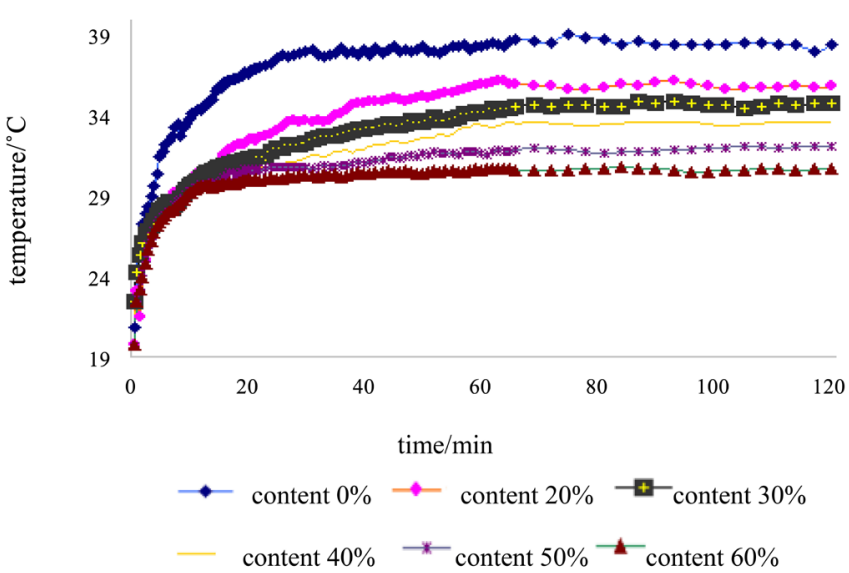

Figure 7. The effect of gas gel content on heat insulation. 


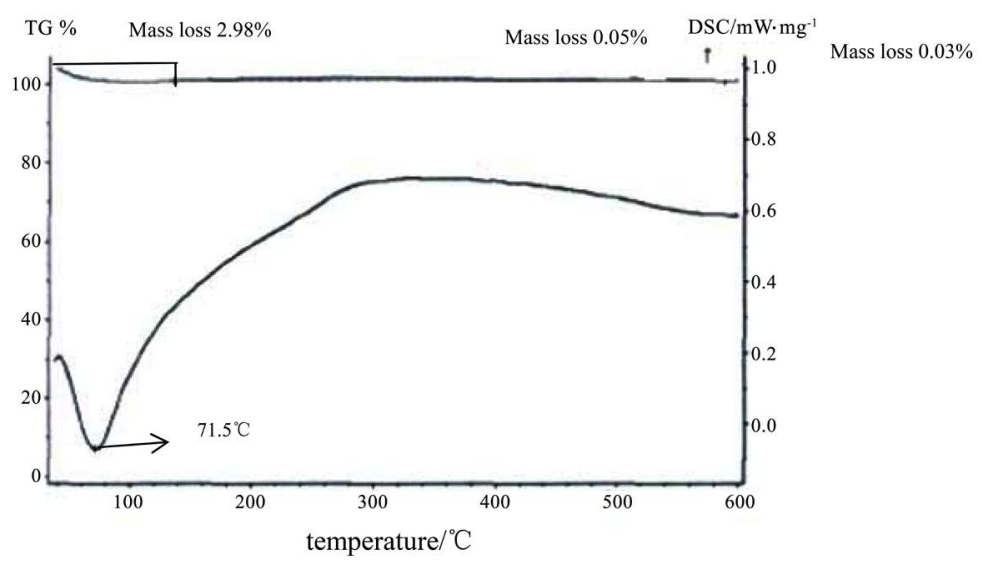

Figure 8. The DSC - TG curve of the composite material.

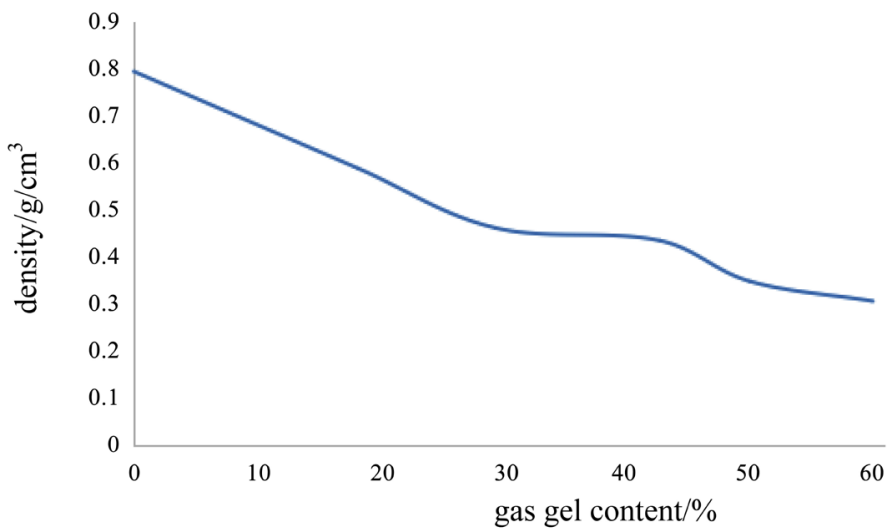

Figure 9. The effect of gas gel content on the density of composite material.

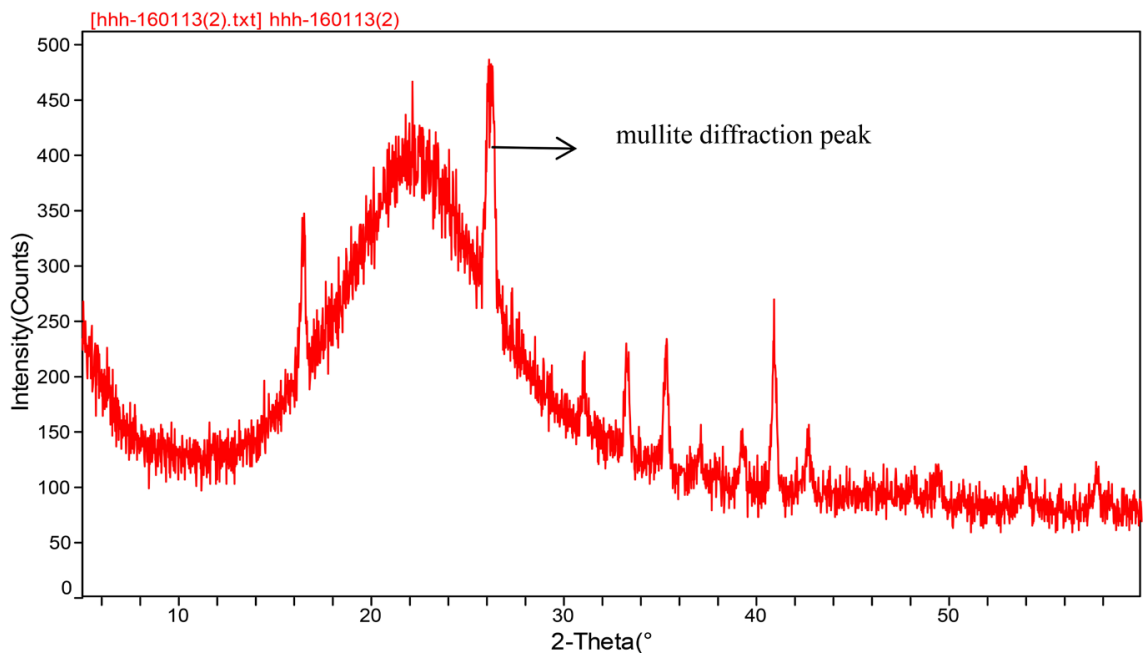

Figure 10. The XRD of heat-insulating composite material (gas gel content $60 \%$ ).

\section{Conclusion}

In sum, it can be seen that at the micron scale the silica aerogel is coated with mullite fiber and filled in the pore of the mullite fiber from the SEM picture. The mullite fibre also played toughening effect in silica gel drying process. Even if the 
gas gel cracked the mullite fibre still kept the macro block structure of the composite material. Therefore the actual application value of the composite material improved. The composite material plate has the effect of heat insulation. The aerogel content was higher, the insulation effect was better. The composite material was stable at $600^{\circ} \mathrm{C}$. With the increasing of silica aerogel content, the density of the composite material was smaller.

\section{Conflicts of Interest}

The authors declare no conflicts of interest regarding the publication of this paper.

\section{References}

[1] Qin, H.Y. (2013) Research Progress of Silica Aerogel Materials. Science \& Technology Association Forum, 40-43.

[2] Li, P. (2014) Preparation and Properties of Silica Aerogel Particles. Chang'an University, Xian, 4-6.

[3] Dong, Z.J., Li, X.K. and Yuan, G.M. (2006) Mo Mullite Fiber Enhanced $\mathrm{SiO}_{2}$ Aerogel Composite Material Preparation and Performance. New Chemical Materials, No. 7, 58-61.

[4] Li, T. (2011) Study on the Preparation of Low Thermal Conductivity Insulation Materials. Wuhan University of Technology, Wuhan, 9-10.

[5] Wang, F. (2009) Silica Aerogels Hydrophobic Modified and Ambient Pressure Dying. Beijing University of Chemical Technology, Beijing, 7-12.

[6] Wu, G.Y., Cheng, X., Yu, Y.X., et al. (2010) Preparation of Silica Aerogels via Ambient Pressure Drying. Progress in Chemistry, 22, 1892-1900.

[7] Yang, K., Pang, J.W., Wu, B.R., et al. (2009) Research Progress on Modification Methods and Research Progress of Silica Gel. Transactions of Beijing Institute of Technology, No. 9, 833-837.

[8] Sun, D., Zhou, C.L., Chen, H., et al. (2015) Research Status and Application Prospect of Silica Gel. Advanced Ceramics, No. 4, 24-31.

[9] Rao, A.V. and Kulkarni, M. (2002) Hydrophobic Properties of TMOS/TMES-Based Silica Aerogels. Materials Reasear Bulletin, 37, 1667-1677. https://doi.org/10.1016/S0025-5408(02)00795-X

[10] Wang, F., Li, R.F. and Luo, Z.K. (2010) Preparation of Mesoporous $\mathrm{SiO}_{2}$ by Normal Pressure Drying Method. Journal of Shenzhen University Science and Engineering, No. 1, 70-75.

[11] Yao, S.Y. (2006) Preparing Method of Continuous Mullite Fibers. China Academy of Mechinery Science and Technology, Beijing, 6-9.

[12] Wu, Z. (2010) Preparation and Characterization of Silica Aerogels by Supercritical Drying Technique. Changchun University of Science and Technology, Changchun, 22-26.

[13] Tian, H., Chen, L.X. and Liu, Q.W. (2007) The Properties, Preparation and Application of Silica Sol. Science and Technology of Overseas Building Materials, No. 2, $8-11$.

[14] Fan, Z.T., Wang, J.N., Wang, H.F., et al. (2007) Present Situation and Development trend of Water Glass Binder Modification Technology. Modern Cast Iron, No. 04, 
76-80.

[15] He, Y. (2005) Rapid Thermal Conductivity Measurement with a Hot Disk Sensor: Part 2. Characterization of Thermal Greases. Thermochimica Acta, 436, 130-134. https://doi.org/10.1016/j.tca.2005.07.003 